Chinese American older adults, comparing Mandarin and Cantonese speakers on: preferences for life-sustaining treatment at EOL; communication with family and physician about these preferences; knowledge, attitudes and behaviors toward advance directives and hospice care. Reasons for the language group differences, as well as lack of differences, are discussed.

COMPARING MANDARIN- AND CANTONESESPEAKING OLDER ADULTS: END-OF-LIFE CARE KNOWLEDGE, ATTITUDES, AND BEHAVIORS

C. Berkman ${ }^{1}$, X. Liu' 2 1. Fordham University, New York, New York, United States, 2. MSW Fordham University Graduate School of Social Service, New York, NY, USA

Most studies of racial/ethnic differences on knowledge, attitudes, and behaviors toward end-of-life (EOL) care treat Asians as one group. We compared two Chinese subgroups, Mandarin $(\mathrm{n}=150)$ and Cantonese $(\mathrm{n}=112)$ speakers. There was no difference in knowledge about living wills (LWs). Mandarin speakers had more knowledge about health care proxies (HCPs) $(\mathrm{p}=.03)$ and more positive attitudes toward LWs $(\mathrm{p}=.01)$ and HCPs $(\mathrm{p}=.02)$. Completion of both HCPs and LWs was low and not different by language. Mandarin speakers were more likely to want life-sustaining treatment at EOL $(\mathrm{p}<.001)$, including if in a permanent coma $(\mathrm{p}<.01)$. Cantonese speakers were more likely to want pain medication, even if it shortened their life $(p=p<.01)$. There were no differences in preferences for communication with family or physician about diagnosis, prognosis, and EOL treatment. Mandarin speakers were more likely to want to discuss EOL treatment preferences with family $(\mathrm{p}<.01)$. Reasons and implications of findings are discussed.

\section{END-OF-LIFE CONVERSATIONS AND HOSPICE PLACEMENT: ASSOCIATION WITH LESS AGGRESSIVE CARE DESIRED IN THE NURSING HOME}

J.P. Reinhardt ${ }^{1}$, D. Downes ${ }^{2}$, V. Cimarolli ${ }^{3}$, P. Bomba ${ }^{4}, 1$. The New Jewish Home, New York, New York, United States, 2. MSW Isabella Geriatric Center, New York, New York, USA, 3. PhD Research Institute on Aging, The New Jewish Home, New York, New York, USA, 4. MD Excellus BlueCross BlueShield \& MedAmerica Insurance Company, Rochester, New York, USA

Education about end-of-life care (EOL) and treatment options are important for older adult nursing home residents with chronic, progressive decline and end-stage disease. Research has shown that racial/ethnic disparities persist in EOL care with ethnic minorities being less likely to reverse the default of aggressive care. This study used retrospective data from a sample of racially/ethnically diverse long-stay nursing home decedents ( $\mathrm{N}=297 ; 48 \%$ non-Hispanic white, $31 \%$ black, $21 \%$ Hispanic) taken from electronic health records to capture the end-of-life experience. Findings showed no significant differences among the three groups regarding treatment discussions, medical orders, distressing symptoms, and treatments received in the 6 months before death. Only discussion about intubation and having a Do Not Intubate order differed, with Black and Hispanic decedents more likely than White to have discussed this, and to have a DNI order in place. Perhaps nursing home residence minimized differences in EOL care by race/ethnicity.

\section{RACIAL AND ETHNIC DIFFERENCES IN HOSPICE} USE: A SECONDARY ANALYSIS OF THE HEALTH AND RETIREMENT STUDY (HRS)

J. Han, Fordham University Graduate School of Social

Service, New York, New York, United States

This study examined race and ethnic differences in hospice use. The Health and Retirement Study 2014 wave, a nationally representative sample was used. African Americans $(\mathrm{n}=128)$ and Hispanics $(\mathrm{n}=71)$ were oversamples. We examined the 922 decedents who had died at age 65 or older and had an exit interview completed by a proxy. Hospice was used by $52.3 \%$ of white non-Hispanic, $49.2 \%$ of African American, and $42.3 \%$ of Hispanic decedents. This difference was not significant, a finding that is inconsistent with most previous studies. Reasons for lack of a race and ethnic difference in this study are discussed. Health care providers should be aware of that regardless of race and ethnicity, hospice utilizations rates remain low. Research is needed to identify factors that increase hospice use.

\section{ATTITUDES TOWARD HOSPICE CARE:} A COMPARISON OF CANTONESE- AND MANDARINSPEAKING CHINESE AMERICAN OLDER ADULTS

X. Liu ${ }^{1}$, C. Berkman ${ }^{2}$ 1. Fordham University Graduate School of Social Service, New York, New York, United States, 2. PhD Fordham University Graduate School of Social Service, New York, NY, USA

The study examined knowledge, attitudes, and preference for hospice care comparing among 262 Chinese American senior center members. Interviews were conducted in Mandarin $(57.3 \%)$ and Cantonese $(42.7 \%)$. Measures included a five-item scale to measure knowledge, a 13-item scale to measure attitudes, and a single question about whether the senior would want hospice care if near the end of life. Cantonese speakers had more positive attitudes toward hospice care than Mandarin speakers, when controlling for sociodemographic characteristics, health status, religiosity and acculturation, Cantonese speakers had more a positive attitude toward hospice care than Mandarin speakers $(\mathrm{p}=.02)$. Despite this, there was no difference by language in respondents would want hospice care by themselves. There were also no differences by language group in knowledge about hospice. Reasons for the differences - and lack of difference - by language group will be discussed.

\section{SESSION 1415 (SYMPOSIUM)}

\section{EMPOWERING OLDER ADULTS: OPPORTUNITIES AND CHALLENGES}

Chair: A. Glicksman, Philadelphia Corporation for Aging, Philadelphia, Pennsylvania

Empowering older adults so that they can make their own decisions regarding where and how they live is a frequently stated goal that can be difficult to achieve. Programs designed to serve older adults are often limited by regulations, resources, and tension between the desires of an older adult and what seems safest and healthiest in the eyes of the 